\title{
Analysis of Various Organic and Organometallic Compounds Using Nanostructure-Assisted Laser Desorption/Ionization Time-of-Flight Mass Spectrometry (NALDI-TOFMS)
}

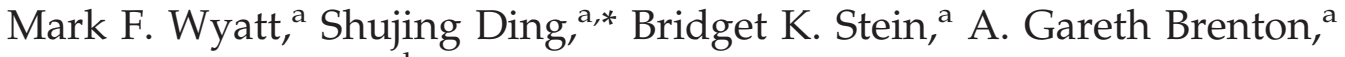 \\ and R. Hugh Daniels ${ }^{b}$ \\ ${ }^{a}$ EPSRC National Mass Spectrometry Service Centre (NMSSC), Institute of Mass Spectrometry (IMS), School \\ of Medicine, Swansea University, Swansea, United Kingdom \\ ${ }^{\mathrm{b}}$ Nanosys Inc., Palo Alto, California, USA
}

\begin{abstract}
Nanostructure-assisted laser desorption/ionization time-of-flight mass spectrometry (NALDITOFMS) has been developed recently as a matrix-free/surface-assisted alternative to matrix-assisted laser desorption/ionization time-of-flight mass spectrometry (MALDITOFMS). The NALDI surface of silicon nanowires is already very effective for the analysis of small to medium sized, polar organic molecules in positive ion mode. The current study examined this technology for the analysis of several nonpolar organic, organometallic, and ionic compounds in positive ion mode, as well as a fluorinated compound and various acids in negative ion mode. NALDI data are compared and contrasted with MALDI data for the same compounds, and the higher sensitivity of NALDI is highlighted by the successful characterization of two porphyrins for a sample amount of 10 amol per spot. (J Am Soc Mass Spectrom 2010, 21, 1256-1259) (c) 2010 American Society for Mass Spectrometry
\end{abstract}

$\mathrm{T}$ The established matrix-assisted laser desorption/ ionization time-of-flight mass spectrometry (MALDITOFMS) technique of co-crystallizing an analyte with a radiation absorbing matrix is not optimal for the analysis of small molecules $(<600 \mathrm{Da})$ due to high intensity matrix species that dominate the spectrum in the low mass range. The large choice of matrix compounds with varying properties also means that sample preparation is problematic and requires analyst expertise to achieve reproducible data. To address both of these problems, several research groups have investigated matrix-free or surface-assisted techniques, that is, the analyte does not require mixing and co-crystallization with any matrix compound. The aim is to produce an immobilized surface that mimics the matrix, but would give little or no background ions, while also removing the requirement for analyte/matrix mixing. Deposition of an analyte so-

Address reprint requests to Dr. M. F. Wyatt, EPSRC National Mass Spectrometry Service Centre (NMSSC), Institute of Mass Spectrometry (IMS), School of Medicine, Swansea University, Singleton Park, Swansea SA2 8PP, UK. E-mail: m.f.wyatt@swansea.ac.uk

* Current address: Proteomics Group, MRC Mitochondrial Biology Unit, Wellcome Trust/MRC Building, Hills Road, Cambridge CB2 0XY, UK. lution directly onto a surface would greatly simplify sample preparation.

Tanaka's pioneering work with cobalt particles dispersed in glycerol [1] has been followed by studies of other metal and metal oxide particles [2], but these methods are better termed as using an 'inorganic matrix' rather than being surface-assisted. The first surface-assisted method to show promise was the use of graphite particles [3], which afforded successful characterization of several peptides. The development of desorptionionization on a random porous silicon surface (DIOS) [4], not only allowed surface-assisted analysis of peptides, but also afforded spectra of small organic molecules devoid of matrix species. A more ordered silicon nanocavity array has been fabricated using nanosphere lithography [5]. Matrix-free techniques were reviewed in detail in 2007 by Peterson [6]. The area has continued to develop, with several silicon-based variations, including unmodified silica [7], modified silica gels (mfMELDI-MS) [8], silicon nitride nanoparticles [9], and chemically modified porous silicon [10].

One variation that has given very encouraging results is desorption-ionization on silicon nanowires [11, 12], grown from silane vapor, which has recently been commercialized as nanostructure-assisted laser desorption/ 
ionization (NALDI) [13]. The term NALDI has also been coined to describe other forms of nanowire surface [14]. Silicon nanowires have also recently been prepared via chemical etching [15]. NALDI surfaces are applied directly onto standard stainless steel target plates and provide a robust, stable surface that can be used in standard instrument configurations. The NALDI surface has been evaluated for over forty mostly polar organic compounds [16], and has been directly compared to the performance of pencil lead, silica gel, and DIOS surfaces in analyzing over fifty peptides [17].

This present study has focused on the application of NALDI to a range of non-polar organic compounds, organometallic and coordination complexes, which are compounds we routinely analyze by MALDI. These compounds and ionic analytes were analyzed in positive ion mode, whilst a highly fluorinated compound and analytes containing various acid functionalities employed negative ion mode.

\section{Experimental}

Dithranol, meso-tetraphenylporphyrin (TPP), mesotetra-4-methylphenylporphyrin (TMPP), meso-tetra (pentafluorophenyl)porphyrin $\left(\mathrm{TF}_{5} \mathrm{PP}\right)$, copper nitrate $\left(\mathrm{Cu}\left(\mathrm{NO}_{3}\right)_{2}\right)$, silver nitrate $\left(\mathrm{AgNO}_{3}\right)$, polystyrene (PS, $\left.M_{\mathrm{n}}=600\right)$, and various transition-metal acetylacetonate (acac) complexes (Fe(II), Co(II), Ni(II), Cu(II), Zn(II), V(III), $\mathrm{Cr}(\mathrm{III}), \mathrm{Mn}(\mathrm{III}), \mathrm{Fe}(\mathrm{III})$, and $\mathrm{Co}(\mathrm{III}))$ were purchased from Sigma-Aldrich (Dorset, UK). The remaining analytes (see Acknowledgements for details of researchers whose samples have been freely provided and characterized in this study) were submitted for analysis to the EPSRC National Mass Spectrometry Service Centre (NMSSC). 2-[(2E)-3-(4tert-butylphenyl)-2-methylprop-2-enylidene] malononitrile (DCTB) matrix, 1,1,1,3,3,3-hexafluoro-2-propanol (HFIP, $\geq 99 \%$ ) and tetrahydrofuran (THF, $\geq 99.5 \%$ ) were purchased from Fluka (Dorset, UK). HPLC grade dichloromethane (DCM) and methanol $(\mathrm{MeOH})$ were purchased from Fischer Scientific (Loughborough, UK).

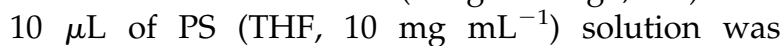
vortex-mixed with $49 \mu \mathrm{L}$ of THF, and with $0.5 \mu \mathrm{L}$ of $\mathrm{Cu}\left(\mathrm{NO}_{3}\right)_{2}\left(\mathrm{MeOH}, 10 \mathrm{mg} \mathrm{mL}^{-1}\right)$ or $\mathrm{AgNO}_{3}(\mathrm{THF}, 10 \mathrm{mg}$ $\left.\mathrm{mL}^{-1}\right)$. For comparative MALDI analysis, $49 \mu \mathrm{L}$ of dithranol (THF, $20 \mathrm{mg} \mathrm{mL}^{-1}$ ) was used. For the remaining analytes (acac complexes in HFIP, $\sim 1 \mathrm{mg} \mathrm{mL}^{-1}$, except V(III) complex and all other analytes in DCM, 1 $\left.\mathrm{mg} \mathrm{mL}{ }^{-1}\right), 1 \mu \mathrm{L}$ of analyte solution was vortex-mixed with $49 \mu \mathrm{L}$ of the same solvent, with $49 \mu \mathrm{L}$ of DCTB (DCM, $20 \mathrm{mg} \mathrm{mL}^{-1}$ ) being used for comparative MALDI analyses; $0.5 \mu \mathrm{L}$ of the final mixture was spotted onto a NALDI target or a gold-plated, deepwelled MALDI plate.

NALDI and MALDI-TOFMS spectra were acquired using an Applied Biosystems Voyager DE-STR spectrometer (Framingham, MA, USA), operated in positive and negative ion, reflectron mode; an Opti-TOF plate holder allowed entry of the NALDI target into the spectrometer.

\section{Results and Discussion}

The qualitative investigations began with the analysis of aryl functionalized porphyrins TPP and TMPP. It is extremely advantageous that due to the hydrophobic properties of the NALDI surface even samples in the most volatile of solvents (such as DCM in this case) do not spread, allowing discrete and easy sample deposition, and preventing contamination of adjacent wells. Interestingly, the $[\mathrm{M}+\mathrm{H}]^{+}$species was observed for both porphyrins, in contrast to the positive radical $\left(\mathrm{M}^{+\cdot}\right)$ species typically observed in MALDI.

To test the sensitivity of the NALDI surface relative to MALDI, both porphyrin solutions were successively diluted by a factor of 10 and re-analyzed. MALDI spectra of the analyte species with good signal-tonoise $(S / N \geq 10: 1)$ were acquired for $10 \mathrm{fmol}$ of analyte per deposition spot, whilst the NALDI surface gave similar $\mathrm{S} / \mathrm{N}$ ratios down to $\sim 10$ amol of analyte per deposition spot (see Figure 1). These values represent a 1000 times typical increase in sensitivity for the NALDI surface, which are comparable to the findings of other researchers [11, 16, 17].

The fluorinated porphyrin $\mathrm{TF}_{5} \mathrm{PP}$ was studied, for which the $\mathrm{M}^{+\cdot}$ species is not usually observed by MALDI. However, a mixture of $\mathrm{M}^{+\cdot}$ and $[\mathrm{M}+\mathrm{H}]^{+}$as well as fragment species were observed using NALDI (see Supplementary Material, Figure S1, which can be found in the electronic version of this article), and the ion intensity is much lower than the non-fluorinated porphyrin data acquired under similar conditions. An-

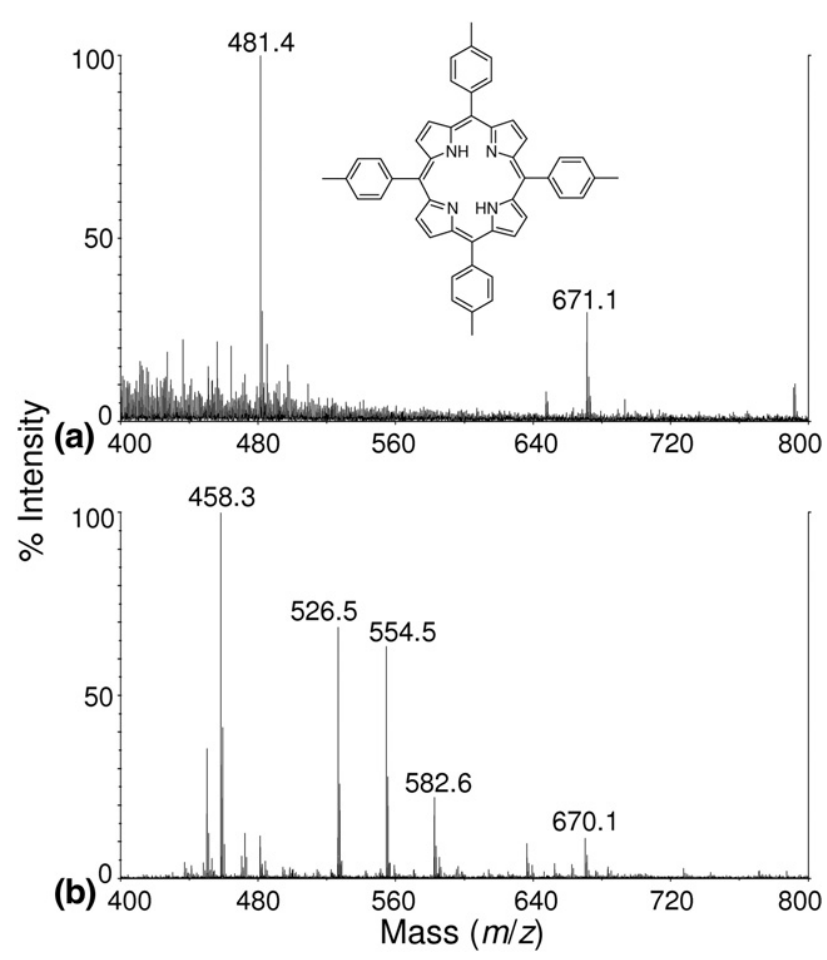

Figure 1. Sensitivity comparison of TMPP spectra: (a) 10 amol by NALDI $\left([\mathrm{M}+\mathrm{H}]^{+}\right.$species $\left.\mathrm{m} / \mathrm{z} 671\right)$, and (b) $10 \mathrm{fmol}$ by MALDI (DCTB; $\mathrm{M}^{+\cdot}$ species $m / z 670$ ). 
other porphyrin studied had a zinc atom coordinated within the cavity of the macrocycle $(M W=910)$ and would normally give the $\mathrm{M}^{+\cdot}$ species by MALDI. However, an apparent overlapping mixture of the $[\mathrm{M}-\mathrm{H}]^{+}$ and $\mathrm{M}^{+\cdot}$ species was observed by NALDI (see Supplementary Material, Figure S2). The porphyrins considered previously were all free base compounds capable of being protonated, but here this ionization route is blocked by the coordinated zinc atom, so ionization pathways usually considered unfavorable are now utilized. To examine this theory further, an aryl-boron compound ( $\mathrm{MW}=838$ ) without obvious protonation sites was studied. In MALDI, this compound simply gives the $\mathrm{M}^{+\cdot}$ species, and the same species is the base peak observed using NALDI with the addition of fragmentation ions (see Supplementary Material, Figure S3), which indicates that the NALDI surface can be a harder ionization technique than MALDI, depending upon the analyte.

Oligomeric PS was investigated, and the lack of conjugation between each aryl group per repeating unit means the $\mathrm{M}^{+}$species is not normally observed by MALDI; instead copper or silver cationizing agents are added to observe the PS distribution. $[\mathrm{M}+\mathrm{Na} / \mathrm{K}]^{+}$ species for polar analytes have been noted previously in NALDI by relying on ubiquitous sources of metal ions $[16,17]$. In the equivalent NALDI analyses, the [M + $\mathrm{Cu}]^{+}$PS distribution was easily acquired (see Figure 2), but the analogous $[\mathrm{M}+\mathrm{Ag}]^{+}$PS distribution was not observed. Possibly the NALDI surface has a high affinity for $\mathrm{Ag}^{+}$ions, so they are not desorbed with typical laser fluences and thus unable to undergo gas-phase binding to PS chains and be detected.

Organometallic and coordination complexes were also studied by examining a palladium-ferrocene complex $(\mathrm{MW}=662)$, again typically observed as the $\mathrm{M}^{+}$. species by MALDI. Similar to the aryl-boron compound above, the $\mathrm{M}^{+}$species, along with fragment ions, were observed using the NALDI surface, but these latter species are now the base peak of the spectrum, as shown in Figure 3. The observation can be rationalized by considering the weaker bond strengths of the

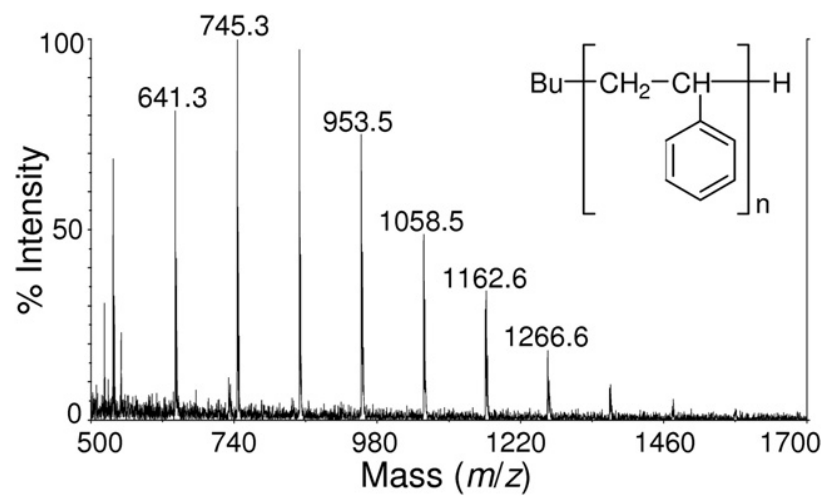

Figure 2. NALDI spectrum showing the $[\mathrm{M}+\mathrm{Cu}]^{+}$PS distribution.

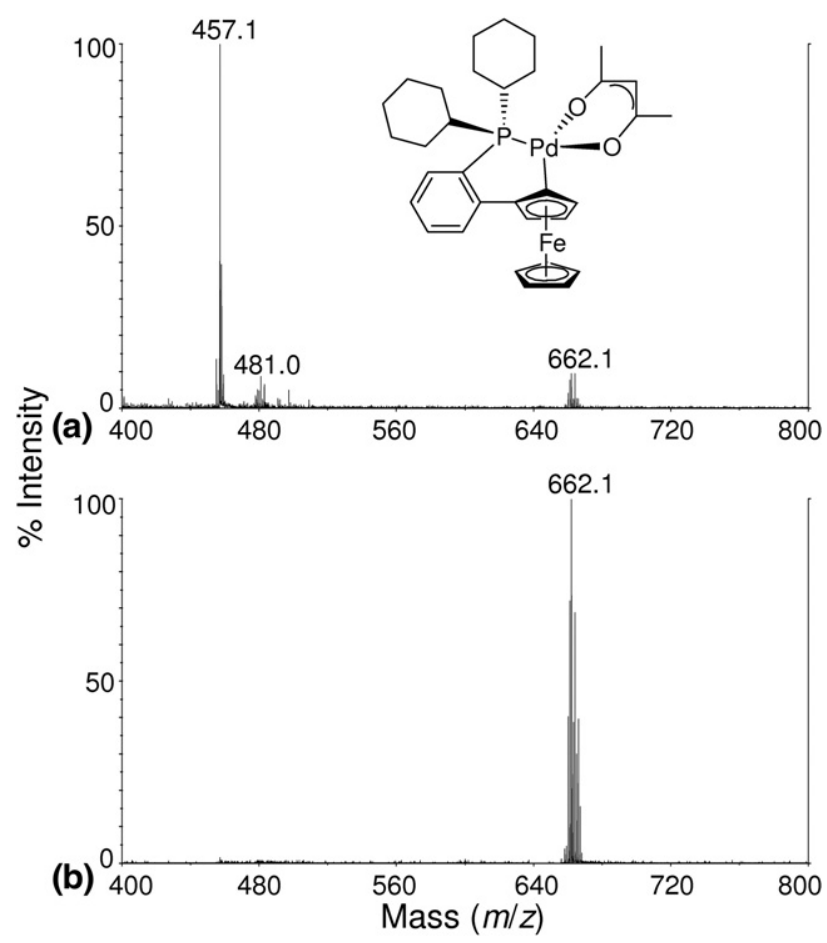

Figure 3. Comparison of spectra for a palladium-ferrocene compound: (a) NALDI, and (b) MALDI (DCTB).

palladium-ferrocene complex, which further indicates that the NALDI surface can be a harder ionization technique under certain circumstances. Similar observations were made between MALDI and NALDI for platinum and iridium coordination complexes (see Supplementary Material, Figures S4 and S5, respectively), but higher mass species were also observed. It is unlikely that the NALDI surface has reacted with the analyte, and more likely that the higher sensitivity and range of ionization mechanisms available simultaneously have led to the observation of impurities within the sample. The ten acetylacetonate complexes have been studied previously by MALDI and their intricate spectra, surprising for such simple compounds, have been discussed in detail [18]. Similar or even more intricate data for these complexes were acquired using the NALDI surface (see Supplementary Material, Figures S6).

The ability of the NALDI surface to form protonated species is well documented, so we examined if the deprotonation mechanism was accessible by studying acidic compounds. Four boronic acids and three compounds with multiple carboxylic and/or sulfonic acid groups were analyzed in negative ion mode, but only fragment ions were observed. A fullerene with two carboxylic acid groups, observed as either $\mathrm{M}^{+\cdot}$ or $\mathrm{M}^{-}$. species in MALDI, also only gave fragment ions in NALDI. However, $\mathrm{TF}_{5} \mathrm{PP}$ was observed as the $\mathrm{M}^{-}$. species in both methods (see Supplementary Material, Figure S1).

Finally, three isomeric hypervalent iodine trifluoroacetate (TFA) salts were investigated in positive ion mode. 
The salts were expected to exist as dimers (MW = 992), for which the $[2 \mathrm{M}-\mathrm{TFA}]^{+}$species was exclusively observed by MALDI, and with only the desorption process being probed, similar results were expected using NALDI. Unlike MALDI, the base peak of the NALDI data were $[\mathrm{M}-\mathrm{TFA}]^{+}$species for the monomer, with the corresponding dimer species observed at very low intensity (see Supplementary Material, Figure S7). Identical data were acquired for all three isomers and these data again suggest the NALDI surface can be a harder ionization technique than MALDI.

\section{Acknowledgments}

The authors thank EPSRC for funding this work (grant no. GR/R70088/01). They also thank Dr. M. A. Carroll, University of Newcastle, Dr. T. D. James, University of Bath, Dr. C. J. Richards, Queen Mary, University of London, Dr. J. A. Weinstein, University of Sheffield, Dr. A. N. Cammidge, University of East Anglia, and Professor T. B. Marder, University of Durham, for permission to reproduce data pertaining to their compounds.

\section{Appendix A Supplementary Material}

Supplementary material associated with this article may be found in the online version at doi:10.1016/ j.jasms.2010.03.038.

\section{References}

1. Tanaka, K.; Waki, H.; Ido, Y.; Akita, S.; Yoshida, Y.; Yoshida, T.; Matsuo, T. Protein and Polymer Analyses up to $m / z 100,000$ by Laser Ionization Time-of-Flight Mass Spectrometry. Rapid Commun. Mass Spectrom. 1988, 2, 151-153.

2. Kinumi, T.; Saisu, T.; Takayama, M.; Niwa, H. Matrix-Assisted Laser Desorption/Ionization Time-of-Flight Mass Spectrometry Using an Inorganic Particle Matrix for Small Molecule Analysis. J. Mass Spectrom. 2000, 35, 417-422.
3. Sunner, J.; Dratz, E.; Chen, Y. C. Graphite Surface Assisted Laser Desorption/Ionization Time-of-Flight Mass-Spectrometry of Peptides and Proteins from Liquid Solutions. Anal. Chem. 1995, 67, 4335-4342.

4. Wei, J.; Buriak, J. M.; Siuzdak, G. Desorption-Ionization Mass Spectrometry on Porous Silicon. Nature 1999, 399, 243-246.

5. Finkel, N. H.; Prevo, B. G.; Velev, O. D.; He, L. Ordered Silicon Nanocavity Arrays in Surface-Assisted Desorption/Ionization Mass Spectrometry. Anal. Chem. 2005, 77, 1088-1095.

6. Peterson, D. S. Matrix-Free Methods for Laser Desorption/Ionization Mass Spectrometry. Mass Spectrom. Rev. 2007, 26, 19-34.

7. Shenar, N.; Martinez, J.; Enjalbal, C. Laser Desorption/Ionization Mass Spectrometry on Porous Silica and Alumina for Peptide Mass Fingerprinting. J. Am. Soc. Mass Spectrom. 2008, 19, 632-644.

8. Feuerstein, I.; Najam-ul-Haq, M.; Rainer, M.; Trojer, L.; Bakry, R.; Aprilita, N. H.; Stecher, G.; Huck, C. W.; Bonn, G. K.; Klocker, H.; Bartsch, G.; Guttman, A. Material-Enhanced Laser Desorption/Ionization (MELDI) —a New Protein Profiling Tool Utilizing Specific Carrier Materials for Time of Flight Mass Spectrometric Analysis. J. Am. Soc. Mass Spectrom. 2006, 17 1203-1208.

9. Shariatgorji, M.; Amini, N.; Ilag, L. L. Silicon Nitride Nanoparticles for Surface-Assisted Laser Desorption/Ionization of Small Molecules. J. Nanopart. Res. 2009, 11, 1509-1512.

10. Shmigol, I. V.; Alekseev, S. A.; Lavrynenko, O. Y.; Vasylieva, N. S.; Zaitsev, V. N.; Barbier, D.; Pokrovsky, V. A. Chemically Modified Porous Silicon for Laser Desorption/Ionization Mass Spectrometry of Ionic Dyes. J. Mass Spectrom. 2009, 44, 1234-1240.

11. Go, E. P.; Apon, J. V.; Luo, G. H.; Saghatelian, A.; Daniels, R. H.; Sahi, V.; Dubrow, R.; Cravatt, B. F.; Vertes, A.; Siuzdak, G. Desorption/ Ionization on Silicon Nanowires. Anal. Chem. 2005, 77, 1641-1646.

12. Luo, G. H.; Chen, Y.; Daniels, H.; Dubrow, R.; Vertes, A. Internal Energy Transfer in Laser Desorption/Ionization from Silicon Nanowires. J. Phys. Chem. B 2006, 110, 13381-13386.

13. Daniels, R. H.; Dikler, S.; Li, E.; Stacey, C. Break Free of the Matrix: Sensitive and Rapid Analysis of Small Molecules Using Nanostructured Surfaces and LDI-TOF Mass Spectrometry. J. Assoc. Lab. Automation 2008, 13, 314-321.

14. Kang, M.-J·; Pyun, J.-C.; Lee, J.-C.; Choi, Y.-J · Park, J.-H.; Park, J.-G.; Lee, J.-G.; Choi, H.-J. Nanowire-Assisted Laser Desorption and Ionization Mass Spectrometry for Quantitative Analysis of Small Molecules. Rapid Commun. Mass Spectrom. 2005, 19, 3166-3170.

15. Piret, G.; Drobecq, H.; Coffinier, Y.; Melnyk, O.; Boukherroub, R. Matrix-Free Laser Desorption/Ionization Mass Spectrometry on Silicon Nanowire Arrays Prepared by Chemical Etching of Crystalline Silicon. Langmuir 2010, 26, 1354-1361.

16. Guenin, E.; Lecouvey, M.; Hardouin, J. Could a Nano-Assisted Laser Desorption/Ionization Target Improve the Study of Small Organic Molecules by Laser Desorption/Ionization Time-of-Flight Mass Spectrometry? Rapid Commun. Mass Spectrom. 2009, 23, 1395-1400.

17. Shenar, N.; Cantel, S.; Martinez, J.; Enjalbal, C. Comparison of Inert Supports in Laser Desorption/Ionization Mass Spectrometry of Peptides: Pencil Lead, Porous Silica Gel, DIOS-chip, and NALDI Target. Rapid Commun. Mass Spectrom. 2009, 23, 2371-2379.

18. Wyatt, M. F.; Havard, S.; Stein, B. K.; Brenton, A. G. Analysis of Transition-Metal Acetylacetonate Complexes by Matrix-Assisted Laser Desorption/Ionization Time-of-Flight Mass Spectrometry. Rapid Commun. Mass Spectrom. 2008, 22, 11-18. 